\title{
Optimization of the Spark Gap Parameters for High Power Ultrasound Applications
}

\author{
M. P. Wilson, L. Balmer*, M. J. Given, S. J. MacGregor, I. V. Timoshkin \\ High Voltage Technologies Group, Institute for Energy and Environment, \\ Department of Electronic \& Electrical Engineering, University of Strathclyde, Glasgow G1 1XW, UK \\ "NRS Technologies Ltd, Unit 4, Mercian Close, Longden Road Industrial Estate, Shrewsbury, Shropshire SY3 9EA, UK
}

\begin{abstract}
There is considerable interest in the industrial and commercial applications of High Power Ultrasound (HPU) generated using pulsed power techniques. These applications include metal peening, the treatment of ores and minerals before extraction, drilling technologies and the comminution and recovery of waste materials. In all of these applications, it is important to optimise the parameters of the discharge causing the shock wave in the working medium to maximise the efficiency of the treatment.

It is possible to measure the intensity of the HPU output at distances relatively far from the discharge through the use of pressure sensors such as pinducers. However, due to the non linear attenuation of the HPU pulse as it passes through the working medium, it is difficult to relate these distant measurements to the behaviour in the active region close to the discharge. Techniques such as ball crusher gauges and Almen strips can be used in this near field region, but interpretation of the measurements is complex.

In a research project at the University of Strathclyde, some applications of HPU to the treatment of waste to assist in recycling have been investigated. Two systems have been considered, slag from the manufacture of stainless steel and bottle glass. With the slag material, it is intended to separate stainless steel from the silicate matrix to permit its recovery. With the bottle glass, the intention is comminution of the material to allow it to be recycled in a more valuable form. Measurements of the efficiency of these processes have been made in terms of the mass of material processed versus the energy input as the parameters of the discharge gap have been varied. In parallel with this work, measurements have been made using pinducer sensors to determine the energy in HPU pulses generated by discharges under identical conditions. Correlations are made between the efficiency of material treatment and the intensity of the HPU pulse measured in the far field. It is hoped that this approach will allow the optimal gap parameters to be determined using pinducer measurements rather than time consuming trials based around materials processing.
\end{abstract}

\section{INTRODUCTION}

There is significant interest in the industrial and commercial applications of High Power Ultrasound (HPU) generated using pulsed power techniques. These applications include metal peening [1], the treatment of ores and minerals before extraction [2], drilling technologies [3], and the comminution and recovery of waste materials. In all of these applications, it is important to optimise the parameters of the discharge causing the shock wave in the working medium in order to maximise the efficiency of the treatment.
In collaboration with NRS Technologies Limited, shock waves have been generated through spark discharges in water at the University of Strathclyde. These shock waves are a wideband source of HPU and this research has been focused on determining the suitability of these HPU sources for the processing of materials for recycling. Two materials have been considered, namely stainless steel slag and bottle glass.

Slag material generated as a by-product of new stainless steel production has been treated. A percentage of stainless steel metal is included in this silicate slag and there is currently no efficient processing method to extract it. Consequently, the slag is stockpiled in landfill sites, awaiting a successful processing technology. HPU provides a novel approach to this problem, allowing the recovery of the valuable stainless steel for recycling. The silicate material is also comminuted into a form suitable for reuse.

There are increasing pressures to recycle bottle glass. However, there is little demand for recycled glass by glass manufacturers. The comminution of glass fragments by HPU treatment produces a uniformly sized material with well rounded edges. In this form, recycled material can have several applications, including filtering systems for water treatment plants. Processing with HPU may provide an economic alternative to current milling techniques, crushing glass particles to the relevant size for reuse in added value applications quickly and efficiently.

Measurements of the effectiveness of these processes have been made in terms of the mass of material processed versus the energy input as the parameters of the HPU source gap have been varied. The parameters investigated were the pulse energy (through varying the charging voltage), the pulse repetition rate and, in the case of stainless steel slag, the interelectrode gap distance.

In parallel with this work, measurements have been made using pinducer sensors to determine the energy in HPU pulses generated by discharges under similar conditions. Correlations are made between the efficiency of material treatment and the intensity of the HPU pulse measured in the far field. It is hoped that this approach will allow the optimal gap parameters to be determined using pinducer measurements, rather than time-consuming trials based around materials processing. 


\section{EXPERIMENTAL METHOD}

Two types of experimental systems have been used for this research. Both types are based around a point-point electrode gap submerged in water. A high voltage (HV) pulse with a sub-microsecond rise-time is applied to the gap, leading to the breakdown of the gap and the production of HPU in the water. The processes involved have been discussed in [4]. The first type of chamber was designed to test the suitability of HPU to comminute waste materials as part of a recycling process. The second chamber is a test facility designed to allow the acoustic output of a HPU source to be monitored.

The material processing chambers are based around a common design [4]. There is an upper chamber which is loaded with the material under test and which contains the electrode system. Below the upper chamber, separated with a perforated sheet with a $45 \%$ open area in the form of $1.7 \mathrm{~mm}$ diameter holes, is a detachable collection chamber to gather comminuted material for analysis. The electrode system is situated vertically at the centre of the chamber. The HV electrode consists of a $5 \mathrm{~mm}$ diameter stainless steel rod encased in a $25 \mathrm{~mm}$ diameter insulating bushing. The earth electrode consists simply of a $10 \mathrm{~mm}$ hexagonal nut, fixed directly onto the perforated sheet. A chamber with a diameter of $155 \mathrm{~mm}$ and a volume of 2.7 litres has been used for results reported in this paper.

The acoustic output of the HPU pulses were studied in a tank of dimensions $2030 \mathrm{~mm}$ (length) $\mathrm{x} 1000 \mathrm{~mm}$ (width) $\mathrm{x}$ $1050 \mathrm{~mm}$ (depth) filled with tap water. This size of tank allowed the acoustic pulses generated by the HPU source to be separated from wall reflections.

The pulsed power supply used in these tests was based around a $60 \mathrm{nF}$ capacitance, driven via a $2.5 \mathrm{~kJ} / \mathrm{s}$ capacitor charging supply and switched via a triggered, $\mathrm{SF}_{6}$-filled spark gap switch. Output voltages of up to $35 \mathrm{kV}$ can be achieved with this supply, allowing a maximum discharge energy of $36.75 \mathrm{~J}$ per pulse. This supply is capable of achieving pulse repetition rates of up to 60pps. The voltage and current waveforms were monitored using a Tektronix P6015A high voltage probe and a Samtech Ltd. DE(CP)-01 resistive current shunt. The acoustic output of the HPU source was measured with a Valpey Fisher VP-1093 pinducer.

\section{RESULTS AND DISCUSSION}

\section{A. Tests on Stainless Steel Slag}

In order to determine the effect on treatment of varying the discharge parameters, samples of stainless steel slag were treated with different charging voltages (and hence pulse energies), pulse repetition rates and inter-electrode gaps. Two different charging voltages were deployed, 30 and $35 \mathrm{kV}$, giving maximum available discharge energies of 27 and $36.75 \mathrm{~J}$ respectively (from $\mathrm{E}=1 / 2 . \mathrm{C} . \mathrm{V}^{2}$ with $\mathrm{C}=60 \mathrm{nF}$ ). Tests were run at pulse repetition rates of 10,20 and 30pps and with inter-electrode gaps of 5 and $10 \mathrm{~mm}$.
The sample mass was held constant at $400 \mathrm{~g}$ for all 12 tests. The sample was placed in the 2.7 litre chamber each time and subjected to 60 seconds of HV pulses to generate HPU. The silicate material which had passed through the grid plate to the lower chamber was then removed to be dried and weighed, before subjecting the sample to a further 60 seconds of HPU treatment. Testing ended here in each case, with a relatively small amount of material being found in the lower chamber. This process allowed for comparison of the treatment efficiency based on the mass of material processed versus the energy input.

From Table I, it can be seen that for constant charging voltage and gap spacing, the silicate collected in the lower chamber after the first minute increases with pulse repetition rate. Comparing the results for different charging voltages with constant $5 \mathrm{~mm}$ gap spacing, it is apparent that the amount of material collected increases with charging voltage (and hence, with pulse energy). The yield per pulse apparently decreases with increasing pulse repetition rate. However, very little silicate is comminuted in the second minute of processing. This suggests that at higher pulse repetition rates, the silicate that can be processed has been exhausted in a period shorter than one minute.

As the gap spacing increases to $10 \mathrm{~mm}$, a drop in the processing efficiency is witnessed. When compared to a $5 \mathrm{~mm}$ gap (with constant charging voltage of $35 \mathrm{kV}$ ), the amount of silicate collected in the first minute is considerably less.

The information in Table I allows for some general predictions for the expected processing rates for this prototype system. A charging voltage of $35 \mathrm{kV}$ produces a treatment rate of $16.62 \mathrm{~kg} / \mathrm{hr}$. It is also possible to determine an approximate value of the energy required to treat one tonne of the silicate material if the process scales linearly - this is $30 \mathrm{kWh}$. Data has been obtained on the quantity of stainless steel recovered from these experiments, approximately $85 \mathrm{~g}$ per sample. However, it is not possible to correlate the mass recovered and the treatment conditions. The authors believe that this may be a result of variations in the steel content of the samples used.

TABLE I

BREAKDOWN OF MATERIALS RECOVERED FOR DIFFERENT GAP PARAMETERS

\begin{tabular}{|c|c|c|c|}
\hline \multirow{2}{*}{$\begin{array}{c}\text { Gap } \\
\text { Voltage }\end{array}$} & $\begin{array}{c}\text { Pulse } \\
\text { Repetition } \\
\text { Rate (pps) }\end{array}$ & After $1^{\text {st }}$ minute & After 2 ${ }^{\text {nd }}$ minute \\
\cline { 2 - 4 } & 10 & 148 & 28 \\
\hline \multirow{2}{*}{$5 \mathrm{~mm} /$} & 20 & 198 & 20 \\
\cline { 2 - 4 } $30 \mathrm{kV}$ & 30 & 204 & 26 \\
\cline { 2 - 4 } & 10 & 176 & 29 \\
\hline \multirow{3}{*}{$5 \mathrm{~mm} /$} & 20 & 252 & 28 \\
\cline { 2 - 4 } $35 \mathrm{kV}$ & 30 & 277 & 30 \\
\cline { 2 - 4 } & 10 & 102 & 30 \\
\hline \multirow{2}{*}{$10 \mathrm{~mm} /$} & 20 & 142 & 25 \\
\cline { 2 - 4 } $35 \mathrm{kV}$ & 30 & 181 & \\
\cline { 2 - 4 } & & & Silicate comminuted (g) \\
\hline
\end{tabular}

\section{B. $\quad$ Tests on Bottle Glass}

In order that a more detailed analysis of the effect of varying the pulse parameters could be conducted, a 
homogenous test material was required. A systematic testing schedule was performed using the 2.7 litre chamber with glass as a test material. Glass was chosen as a highly reproducible sample with a market for recycling.

Charging voltages of $30,32.5$ and $35 \mathrm{kV}$ were utilised to give maximum discharge energies of 27, 31.69 and 36.75J respectively. The pulse repetition rate was varied from 5 to $30 \mathrm{pps}$ in steps of $5 \mathrm{pps}$. In each case, the sample chamber was initially loaded with $1 \mathrm{~kg}$ of bottle glass shards. A standard sample is shown in Fig. 1. The glass shards were obtained by shattering the glass through mechanical impact. Thicker pieces of glass, i.e. the neck and base, were removed before the sample was placed in the treatment chamber. The average size of the shards was of the order of $20 \mathrm{~mm}$.

The material in the treatment chamber was subjected to 60 seconds of HV pulses to generate HPU. Any material in the collection chamber was then removed for drying and weighing, with the material remaining in the confinement chamber subsequently treated for a further 60 seconds.
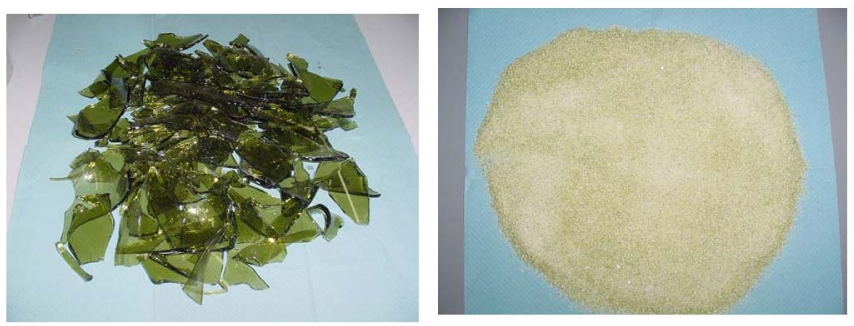

Fig. 1. Glass shards before and after treatment with HPU.

This cycle was repeated for 300 seconds in total (i.e. 5 runs) or until the majority of the initial material had passed through the perforated plate to the collection chamber.

After treatment the glass particle size had been reduced to less than $1.7 \mathrm{~mm}$. A rounding process had also taken place and the typical appearance of the glass after processing is shown in Fig. 1. The cumulative mass of glass processed as the pulse repetition rate was varied between 5 and $30 \mathrm{pps}$ is shown in Fig. 2.

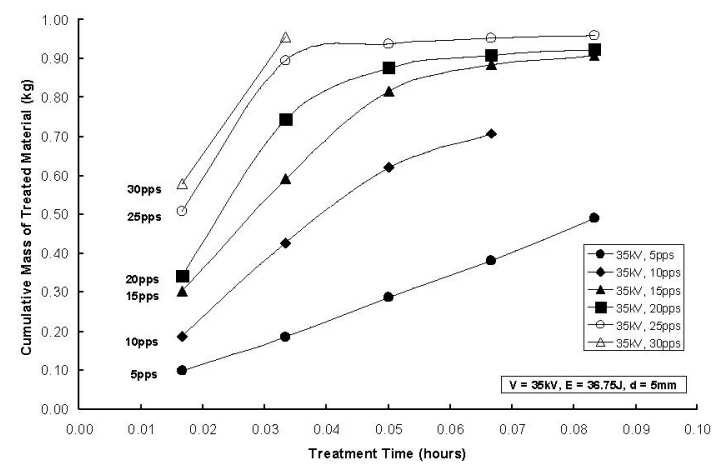

Fig.2. Mass of glass treated as a function of time. Charging voltage $=35 \mathrm{kV}$.

The pulse voltage was $35 \mathrm{kV}$, leading to a maximum available electrical pulse energy of $36.75 \mathrm{~J}$. At the lowest pulse repetition rate, 5pps, the mass of material treated increased linearly with time. As the pulse repetition rate is raised, the rate at which the material is treated increases and evidence of a saturation effect at longer time periods becomes clearly evident. The linear relationship between the mass of glass processed and time of treatment observed for the lower repetition rates suggests that the mass of glass processed depends purely on the number of pulses applied to the system, and consequently, the number of HPU events which have occurred.

The saturation effect observed at the higher repetition rates appears to occur when between 90 and $95 \%$ of the glass originally in the chamber has been treated. At this point, the treatment chamber is almost empty. If attrition processes due to collisions between glass particles are significant in the comminution process, the probability of collisions between glass fragments will decrease as the quantity of the material in the chamber decreases. How significant collisions between glass particles are to the processing rate for the material is presently unknown.

Fig. 3 shows the relationship between the rate at which glass was processed in the first minute as a function of pulse energy and repetition rate. This time interval is used as it is assumed that the processing rate would be relatively constant. It can be seen that there is a linear dependence between the treatment rate and the pulse repetition rate. As the pulse energy was increased by increasing the charging voltage, the treatment rate also increased.

Analysis of the data obtained from these experiments indicated that while the treatment rate varied with pulse repetition rate and pulse energy, the energy required to treat a fixed mass of glass was constant [4].

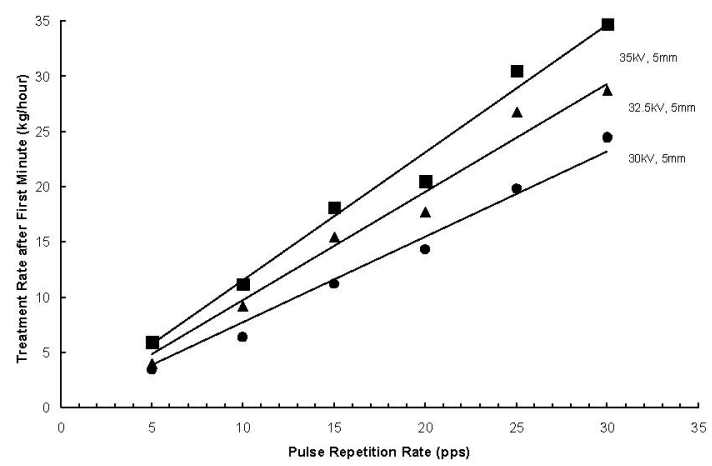

Fig. 3. Initial treatment rate as a function of pulse energy and pulse repetition rate.

\section{C. $\quad$ Pinducer Measurements}

Experiments were performed to see if the behaviour of the acoustic wave relatively far from the HPU source varied in a similar way to the treatment rate data shown in Fig. 3. If this was the case, pinducer measurements could be used to optimise the gap and supply parameters.

The same power supply was used to drive the spark gap in the acoustic test tank, and a gap separation of $5 \mathrm{~mm}$ was 


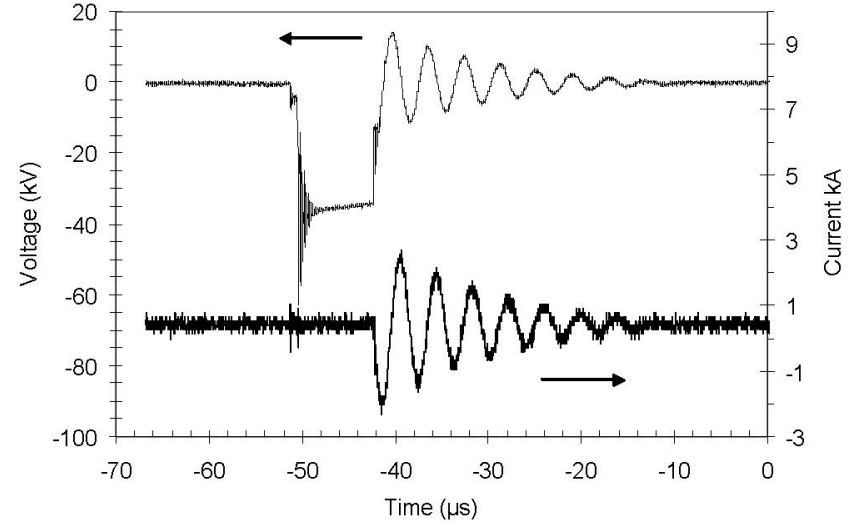

Fig. 4. Typical voltage and current waveforms obtained in acoustic tank

selected. Typical voltage and current waveforms obtained for a charging voltage of $35 \mathrm{kV}$ are shown in Fig. 4.

The form of the pinducer signal observed is shown in Fig. 5 and is similar to that reported in earlier work for much higher pulse energies [5]. Various parameters were extracted from a sample of the pinducer traces; these

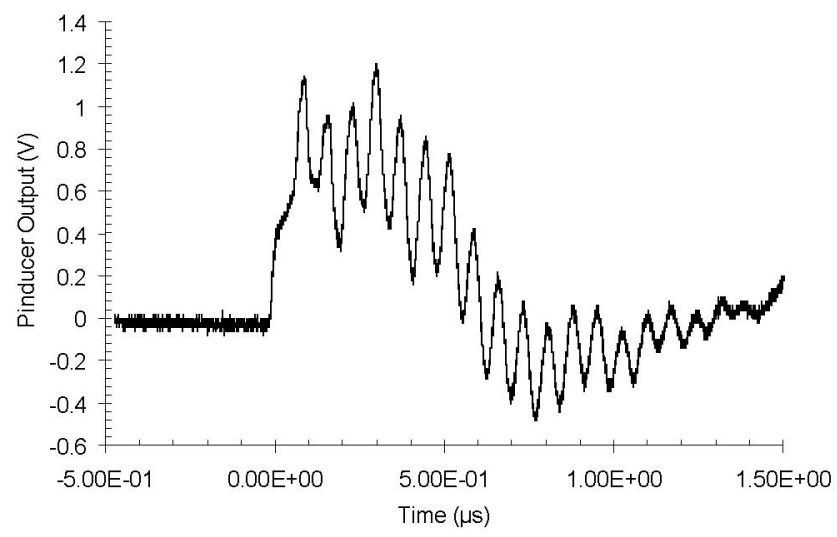

Fig. 5. Pinducer output obtained for waveforms above at a distance of $0.3 \mathrm{~m}$ from the HPU source.

included the magnitude of the initial peak, the maximum peak magnitude, the average of the signal magnitude, and the integral of the signal. The measured parameters were found to be strongly correlated so the signal maximum was used as the parameter of choice. Fig. 6 shows a plot of the energy available to the source at breakdown versus pinducer output for measurements taken at distances of $0.23,0.3$ and $0.5 \mathrm{~m}$ from the source.

It can be seen that as the pinducer is brought closer to the source, the signal magnitude observed on the pinducer increases. However, the correlation between pinducer output and power available to the HPU source is weak, particularly for the measurements at $0.5 \mathrm{~m}$

The vertical lines $\mathrm{a}$ and $\mathrm{b}$ in Fig. 6, correspond to the average available energies for charging voltages of 32.5 and $35 \mathrm{kV}$. For the measurements performed at $0.23 \mathrm{~m}$, there is a change in the estimated pinducer output of approximately $8 \%$.
Observing the equivalent points on Fig. 3, changes of between 30 and $50 \%$ are observed in the initial treatment rate for a corresponding shift in charging voltage. As the pinducer measurements were more sensitive to changes in pulse energy, measurements at a closer range were attempted with the pinducer moved to within $0.1 \mathrm{~m}$ of the HPU source. However, the noise induced on the pinducer circuit by the discharge made it impossible to resolve the pinducer signal.

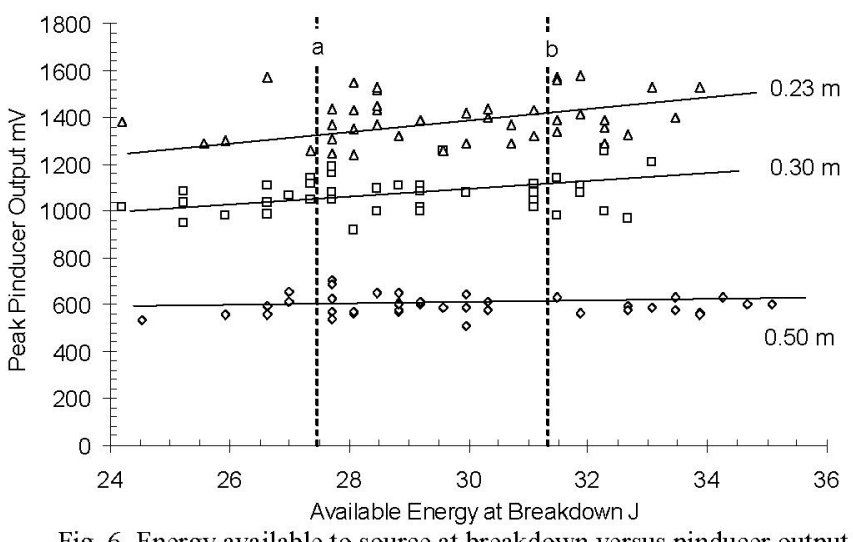

Fig. 6. Energy available to source at breakdown versus pinducer output

\section{CONCLUSIONS}

The results show that pulsed power generated High Power Ultrasound is effective as a method of comminuting both stainless steel slag and bottle glass for recycling. The energy available to a pulse generated HPU source has a marked effect on its behaviour in the region close to the electrodes as shown by the variation of material processing rate with power supply voltage. However, measurements using pinducer sensors in the far field do not reflect the changes observed as the available pulse energy is changed. This suggests that pinducer measurements will not be useful as a means of predicting the efficiency of a HPU source used for materials processing for energies below $50 \mathrm{~J}$.

\section{REFERENCES}

[1] H. Chen, J.W. Kysar, and Y.L. Yao, "Characterization of plastic deformation induced by microscale laser shock peening," Journal of Applied Mechanics, Trans. ASME, vol. 71 (5), pp. 713-723, September 2004.

[2] U. Andres, I. Timoshkin, J. Jirestig, and H. Stallknecht, "Liberation of valuable inclusions in ores and slags by electrical pulses," Powder Technology, vol. 114 (1-3), pp. 40-50, January 2001.

[3] I.V. Timoshkin, J.W. Mackersie, and S.J. MacGregor, "Plasma channel miniature hole drilling technology," IEEE Trans. on Plasma Science, vol. 32 (5), pp. 2055-61, October 2004.

[4] M.P. Wilson, L. Balmer, M.J. Given, S.J. MacGregor, J.W. Mackersie, and I.V. Timoshkin, "Application of electric spark generated high power ultrasound to recover ferrous and non-ferrous metals from slag waste," Minerals Engineering, vol. 19 (5), pp. 491-499, April 2006.

[5] J.W. Mackersie, I.V. Timoshkin, and S.J. MacGregor, "Generation of high-power ultrasound by spark discharges in water," IEEE Trans. on Plasma Science, vol. 33 (5), pp. 1715-24, October 2005. 